\title{
Erratum to: The Determining Risk of Vascular Events by Apnea Monitoring (DREAM) study: design, rationale, and methods
}

\author{
Brian B. Koo ${ }^{1,2}$ • Christine Won ${ }^{3,4}$ - Bernardo J. Selim ${ }^{5} \cdot$ Li Qin $^{6} \cdot$ Sangchoon Jeon $^{7}$ • \\ Nancy S. Redeker ${ }^{7}$ - Dawn M. Bravata ${ }^{8,9,10,11,12}$ - Kingman P. Strohl ${ }^{13,14}$. \\ John Concato ${ }^{15,16}$ • Andrey V. Zinchuk ${ }^{3,4}$ • Henry K. Yaggi ${ }^{3,4,17}$
}

Published online: 12 March 2016

(C) Springer-Verlag Berlin Heidelberg 2016

\section{Erratum to: Sleep Breath}

DOI 10.1007/s11325-015-1254-3

The authors regret that Andrey V. Zinchuk was originally omitted from the submitted authorship line; this has now been updated in the published article.

The online version of the original article can be found at doi: http://dx.doi. org/10.1007/s11325-015-1254-3.

Henry K. Yaggi

henry.yaggi@yale.edu

1 Department of Neurology, Yale University, New Haven, CT, USA

2 Department of Neurology, Connecticut Veterans Affairs Health System, West Haven, CT, USA

3 Department of Pulmonary, Critical Care, and Sleep Medicine, Yale University, New Haven, CT, USA

4 Department of Pulmonary, Critical Care, and Sleep Medicine, Connecticut Veterans Affairs Health System, West Haven, CT, USA

5 Department of Pulmonary, Critical Care, and Sleep Medicine, Mayo Clinic, Rochester, MN, USA

6 Department of Biostatistics, Yale School of Public Health, New Haven, CT, USA

7 Division of Acute Care/Health Systems, Yale School of Nursing, New Haven, CT, USA

8 Department of Neurology, Indiana University School of Medicine, Indianapolis, IN, USA
9 VA HSR\&D Center for Health Information and Communication (CHIC), Richard L. Roudebush VA Medical Center, Indianapolis, IN, USA

10 Department of Internal Medicine, Indiana University School of Medicine, Indianapolis, IN, USA

11 Regenstrief Institute, Indianapolis, IN, USA

12 Department of Medicine, Richard L. Roudebush VA Medical Center, Indianapolis, IN, USA

13 Department of Pulmonary, Critical Care, and Sleep Medicine, Case Western Reserve University, Cleveland, OH, USA

14 Department of Pulmonary, Critical Care, and Sleep Medicine, Louis Stokes Veterans Affairs Medical Center, Cleveland, OH, USA

15 Department of Internal Medicine, Yale University, New Haven, CT, USA

16 Clinical Epidemiology Research Center (CERC), Connecticut Veterans Affairs Health System, West Haven, CT, USA

17300 Cedar Street, New Haven, CT 06443, USA 